\title{
Increasing confusion in a man after a fall-_just a head injury?
}

An error occurred in the units for serum creatinine in the online version of this Endgames article by K Birdi and colleagues (BMJ 2011;343:d4806, doi:10.1136/bmj.d4806). The units are correct in the question section, but in the answer section (in the box titled "Diagnostic criteria for the different forms of myeloma") they should be $\mu \mathrm{mol} / \mathrm{L}$ [not $\mathrm{mmol} / \mathrm{L}$ as we published].

Cite this as: BMJ 2011;343:d5842

๑ BMJ Publishing Group Ltd 2011 